\title{
ReHABILITACIÓN ECOLÓGICA, RESERVA THOMAS VAN DER HAMMEN
}

\author{
REstoration ECOLOgy RESERVE THOMAS VAN DER HAMMEN
}

\author{
Elvia Isabel Casas-Matiz* \\ Ximena Andrea Cabezas Porras**
}

Recibido: 7 de julio de 2017

Aceptado: 23 de octubre de 2017

\section{Resumen}

Este acercamiento busca presentar una alterativa de ocupación dentro de la reserva Thomas van der Hammen, y para ello parte de considerar el estado actual de la reserva, no solo de sus modos de ocupación, si no de las formas de conectividad posible entre los diversos ecosistemas que allí se plantean. El artículo presenta algunas de las fases que se alcanzaron dentro del proceso de la investigación, así como las afectaciones de uno de los actuales usos, los viveros; se revisa el impacto que este uso tiene sobre los humedales presentes en la reserva, buscando que la propuesta plantee un proceso de reconversión de las formas de ocupación a fin de reducir la afectación sobre el suelo.

Palabras clave: conectividad, beneficio ambiental, conservación

\begin{abstract}
This approach seeks to submit an alternative of occupation within the Thomas van der Hammen reserve, and for this purpose, comes from the consideration the current situation of the reserve, not only of their modes of occupation but the types of possible connectivities between the diverse ecosystems raised there. The article presents some of the stages in the process of research were reached, as well as the impacts of one of the current uses, the breeding grounds; the effect that this use has on present wetlands in the reserve is revised, seeking that the proposal proposes a restructuring process of the forms of occupation to reduce the impact on the ground.
\end{abstract}

Keywords: connectivity, environmental benefits, conservation

\footnotetext{
* Magíster en Historia, arquitecta. Grupo: Territorio y habitabilidad. Fundación Universidad América, Bogotá, Colombia. ORCID: https://orcid.org/0000-0002-8200-6697. elvia.casas@ investigadores.uamerica.edu.co

** Arquitecta. Grupo: Territorio y habitabilidad Fundación Universidad de América, Bogotá, Colombia. architecxvd@ gmail.com
} 


\section{INTRODUCCIÓN}

Las reservas hacen parte de la categoría de áreas protegidas, en las que también se encuentran las áreas de gestión de vida silvestre, parque natural, área silvestre y áreas de paisaje protegido, aunque también cubre conceptos de áreas conservadas por la comunidad. (Dudley, 2008). El área de estudio de la reserva Tomas van der Hammen, se engloba dentro del concepto de 'reserva ambiental', que se definen como: "Las áreas protegidas siguen siendo la piedra angular de prácticamente todas las estrategias nacionales e internacionales de conservación, ya que además cuentan con el apoyo de gobiernos e instituciones internacionales como el Convenio sobre la Diversidad Biológica" (Dudley, 2008).

Al ser tan variado el espectro de los elementos constituyentes de las reservas, que estas se pueden considerar como espacios de alto valor por su diversidad. Desde la perspectiva del manejo hídrico, en palabras de Talón y Lastra (2008), se pueden clasificar en tres categorías: parques, reservas naturales y áreas marinas protegidas.

La importancia de las reservas no sólo va enfocada al hecho de ser protectoras de ecosistemas valiosos, diversidad de flora y fauna que se conserva en estos lugares, y que benefician a la gran mayoría de habitantes (Dudley, 2008). Además, por la cantidad de especies que habitan en estas zonas, como aves y mamiferos que no sólo son endémicos, si no que en determinadas epocas del año, ingresan gran variedad de especies de aves migratorias.

Aunque las reservas son de gran beneficio por muchos motivos, también son un punto de discordia debido a que tocan intereses y expectativas entre diferentes actores del territorio. Este es el caso de la Reserva Thomas van der Hammen, ubicada en Bogotá, Colombia.

La reserva, lleva el nombre del eminente científico holandés que la propuso a través de una serie de estudios que realizó a finales del siglo XX, con un equipo de colaboradores de diferentes perfiles profesionales. Ellos, bajo la dirección del profesor van der Hammen se encargaron de elaborar los diferentes protocolos del manejo ambiental. En 2011, y gracias al Acuerdo 11 expedido por la Corporación Autónoma Regional de Cundinamarca-CAR, fue declarada Reserva Forestal Regional Productora del Norte de Bogotá D.C., 'Thomas van der Hammen, y se establecieron una serie de especificaciones para su manejo (CAR, 2011).

En 2014, se generó el acuerdo 021 gracias al cual se adoptó el Plan de Manejo Ambiental de la Reserva Forestal Regional Productora del Norte de Bogotá D.C. Thomas van der Hammen. En este acuerdo se destacan diferentes valores ambientales, que permiten evidenciar que funciona a nivel ecológico como una gran área conectora de la estructura ecológica regional, que cuenta con relictos del ecosistema bosque bajo andino; humedales en buen estado, y conforma el hábitat de aves endémicas con importancia para la conservación, y contiene parte de los mejores suelos agropecuarios del país (CAR, 2014).

La reserva cuenta con una amplia biodiversidad, representada especialmente en aves nativas y migratorias, mamíferos y especies de flora, las cuales hacen parte de los hábitats y ecosistemas que la conforman. En la Sabana de Bogotá se registra la presencia de 200 especies de aves, (nativas y migratorias) (Londoño, 2012).

Las aves existentes tanto en las áreas urbanas como en las rurales y naturales, hacen parte de la cadena trófica, y así mismo, hacen parte del ciclo ecológico que se desarrolla dentro de la estructura ecológica principal. Existen diferentes especies de aves nativas en la ciudad, y muchas de ellas habitan o frecuentan la reserva, como, por ejemplo: la tingua bogotana (Rallus semiplumbeus), que se encuentra en peligro de extinción; la garza africana (Bubulcus ibis), el copetón (Zonotrichia capensis), el maicero (Molothrus bonariensis), entre otros. También se encuentran aves migratorias, como las pirangas (Piranga rubra), el arvejero pechirojo (Pheutictus ludovicianus), el tijereto (Tyrannus savana), entre otros (Molina y Osorio, 1995). 
Además, existen varios mamíferos registrados en los humedales de Bogotá y en el bosque de Las Mercedes, pieza clave de la reserva, como por ejemplo la comadreja (Mustela frenata), la zarigüeya (Didelphimorphia sp.), el curí (Cavia porcellus) entre otros (Ruiz, Otero, Ramires y Trespalacioas, 2008).

Todas las especies de aves y mamiferos, necesitan un ecosistema apto para poder sobrevivir, este ecosistema también se encuentra dentro de la reserva, aunque actualmente se está fragmentando. Como también esta el último relicto de bosque nativo de la Sabana de Bogotá: el bosque de Las Mercedes, que contiene una amplia biodiversidad de la flora original de la Sabana de Bogotá, entre esta vegetación vemos: El Cedro (Cedrela montana), el chusque (Nastus chusque), el arrayán (Myrcia cucullata), entre otros, Ruiz, Otero, Ramires y Trespalacioas (2008).

\section{Problemática actual en la Reserva Thomas Van der Hammen}

Es importante subrayar que la Reserva actualmente está bajo el foco de un debate público en lo concerniente a su desempeño socioeconómico y ambiental, principalmente y entre la administración distrital que busca su urbanización en varias comunidades académicas y ambientales que trabajan por su conservación.

Pasaron tres años de la formulación del plan de manejo ambiental, cuando la CAR aprobó y publicó el Plan de Manejo Ambiental para las 1.395 hectáreas de la Reserva Thomas van der Hammen (CAR, 2014). Este plan adoptaba la posición de la salida de los viveros en un plazo de 7 años, debido a que son 159 hectáreas expansivas, las cuales están afectando la conectividad, y utilizando el agua subterránea para la sustentación de sus cultivos.

El objetivo de este artículo, es el de presentar un panorama general de las problemáticas actuales concernientes a la Reserva, y además, la propuesta Intersticio Ecorrelacional que busca dar soluciones a dichas problemáticas.

\section{Modelos de conectividad afectados en la reserva}

Hay que tener en cuenta que existen tres tipos de conectividad identificados dentro del territorio de la Reserva, para concluir cuales son los valores que se deben rescatar: a) la conectividad de $h a ́-$ bitats, que según Remolina, corresponde a un grupo de especies particulares que se juntan en grupos, siendo la conectividad la unión de dichos parches (Remolina y Chisacá, 2007), también hace referencia a una medida que muestra que tan continuos son los diferentes elementos que conforman el paisaje, desde el punto de vista espacial, para así mostrar el funcionamiento del ecosistema (Sepulveda, Moreira y Villarroel, 1997), este tipo de conectividad en la Reserva Thomas van der Hammen, está fragmentado por una amplia zona de viveros, que interfiere en la relacion y conección de los habitats lo cual hace que las especies se vean amenazadas y corran peligro de extinción; b) la conectividad de paisajes donde Remolina habla de que es la conexión de diferentes parches vegetales que puede llegar a tener un paisaje (Remolina y Hurtado, 2012) aun así la conectividad del paisaje se puede definir como puede ser el desplazamiento de especies de un parche a otro, veneficiándose con recursos, (WWF, 2015), con esta información se podría concluir que no hay conexión del paisaje debido a los diferentes usos que los dividen. Cuando continua la conexión de las aguas subterráneas que conecta los humedales, existe un cambio abrupto por contaminación; y

c) la conectividad ecológica, que incluye los procesos de las cadenas tróficas, procesos de disturbios, flujo de nutrientes, flujos hidrogeológicos, donde estas cadenas se interconectan entre sí (Remolina y Chisacá, 2007); también es la capacidad de desplazamiento de especies en el territorio (WWF, 2015). En la reserva, este tipo de conectividad se ha cortado por el avance urbanístico que ha tenido la ciudad, invadiendo el territorio y disturbando su entorno, haciendo que las especies se 
desplacen a otra zona, o se incremente su riesgo de extinción, ya que la mayoría depende de los habitats particulares para subsistir.

Dentro de la reserva no se cuenta con una conectividad estable, en relación a ninguno de los conceptos arriba mencionados, y por su fragmentación, se puede observar un efecto borde que está afectando algunas zonas. Es importante conservar la conectividad para garantizar los desplazamientos de los organismos donde puedan alimentarse, refugiarse, reproducirse o dispersarse. Si no se cuenta con esto, la supervivencia de las diferentes especies se verá amenazada (Gurrutxaga y Lozano, 2006). De la misma manera, combatir el efecto borde que se está evidenciando. Un grupo de investigadores subraya que la existencia de las especies va ligada al tamaño del parche de su hábitat, es decir, entre más pequeño sea el lugar donde habitan más perdida de especies va a tener (Connor, Courtney y Yoder, 2000). Hay diversas maneras para fomentar la conservación de los parches, y para que así, no se pierdan, una de ellas es generando conectividad entre un parche y otro sector que esté fortalecido, así se genera un beneficio común. Esto en el caso del hábitat óptimo y sub óptimo, un hábitat óptimo que puede estar conectado directamente con otro hábitat aportándole beneficios, y este a su vez, al ser un habitat sub-óptimo le contribuirá según lo que gane (Pulliman, 1988).

\section{Metodología}

La investigación se desarrolló en tres fases: en la primera, se realizó la revisión bibliográfica, donde se consultaron diferentes fuentes, relacionadas con la conectividad; la biodiversidad de aves nativas y migratorias; que hace que las aves migratorias vengan en determinado tiempo; los mamíferos; la vegetación; los decretos y el estado legal y normativo de la reserva; sus respectivos usos, y como estos usos la están afectando, cómo es el caso de la zona de viveros. Además, aspectos como el calentamiento global y cómo puede llegar afectar la reserva; el efecto borde; los análisis del estado del agua; la calidad del viento y otros aspectos ambientales y ecológicos. En la segunda, se adelantaron trabajos de campo en la reserva en los que se tomaron evidencias fotográficas, se recorrió la Reserva en tres ocasiones diferentes, también se hizo un análisis presencial para establecer el tipo de transporte que se destaca a ciertas horas en la reserva, por medio de un conteo, uno a uno, durante una hora en tres horarios diferentes. Se visitaron los humedales que están en la reserva, se observó qué tipo de vegetación crece en este sector, se interactuó con personal de la CAR y del Jardín Botánico. También se participó en caminatas de reconocimiento del lugar con otras universidades.

En tercer lugar, se participó en conferencias donde se ha mostrado la evolución de la investigación, compartiendo la información y complementándola con profesionales de varias disciplinas. Y por último se presentó una serie de avances de la propuesta dividida en tres escalas diferentes, la cual abarca el total de la Reserva, una propuesta general de intenciones por manchas, donde se abarca 1900 hectáreas aproximadamente, después se selecciona la zona de mayor afectación., según la investigación, y se limita a un área de 40 hectáreas, diseñando un plan parcial que da resultado a la problemática más evidente de la investigación, que sería el caso de la zona expansiva de viveros, para mostrar un proyecto de densificación de viveros, observando su mejoramiento en la producción de rosas, y el aprovechamiento del agua lluvia para el edificio, así mismo los proyectos de descontaminación de los recursos hídricos, la reforestación entre otros, permitiendo una rehabilitación de la zona más afectada de la reserva, y por último, dentro del plan maestro, se escoge un sector apto para la muestra de diferentes ambientes, que son propicios dentro y fuera de la reserva, permitiendo que exista una mezcla de actores, ayudando a promover la construcción sostenible dentro del ámbito de la arquitectura, entendiendo su entorno y el respeto al medio ambiente. 


\section{Resultados}

De acuerdo al análisis de la investigación, la pérdida de los ecosistemas de aves, mamíferos, plantas, la contaminación del agua, la pérdida del ciclo de producción ambiental, se debe, en gran medida, a la gran producción de rosas en la reserva ocupando aproximadamente 159 hectáreas de viveros, las malas prácticas agrícolas, la tala de árboles, y la necesidad de vivienda que existe dentro de la ciudad (CAR, 2014). Sin embargo, la producción de rosas representa un gran movimiento en los mercados colombianos. Observando que el nivel de contaminación tan alto que es generado por la zona de vivero, la Corporación Autónoma Regional y su formulación de plan de manejo, concluye que es absolutamente necesario retirar los viveros de la Reserva y reubicarlos en otra zona que tenga la vocación productiva. Pero ¿cómo conciliar la producción de rosas dentro de la reserva de forma sostenible? El sistema funcional de viveros está enfocado en la producción constante de rosas dejando a un lado un sistema ambiental ya reconocido como reserva, dejando así de atender sus relaciones de producción con aquellas interacciones sociales y ecológicas ya existentes.

\section{Actores involucrados}

De esta manera, se refiere como actores a todas aquellas entidades políticas, seres vivos y ecosistemas, personas con diversas ocupaciones, entre otros, que participan, participaron o participaran, activamente dentro de la reserva. A continuación, se nombrarán los principales actores, el rol que cumplen dentro de la reserva y las alternativas de mejoramiento en favor de la reserva.

Actores políticos. Son las entidades gubernamentales ambientales como la Corporación Autónoma Regional de Cundinamarca (CAR), el Ministerio de Medio Ambiente y Recursos Naturales, el Jardín Botánico José Celestino Mutis y la Alcaldía Mayor de Bogotá, que si bien, la mayoría son entidades fundadas para la protección ambiental quedan relegadas con respecto a la prioridad de llevar a cabo proyectos sociales por encima del beneficio ambiental.

Actores ambientales. Actualmente la fauna y flora de la reserva se ven afectadas por la falta de conectividad y pérdida de ecosistemas, por la contaminación de los recursos hídricos. Esto cohíbe el ingreso de nuevas especies rompiendo la cadena trófica y perdiendo aún más especies.

Actores humanos. Los campesinos, agricultores, los trabajadores floricultores, los conductores y la comunidad de la localidad de Suba son actores que influyen, directa o indirectamente, sobre la reserva, usando los recursos de manera inconsciente contaminando los humedales y los recursos ambientales.

Grupos interesados. Actualmente en la reserva existen grupos interesados en protegerla. Para estos grupos se establecen convenios para estudiar los diversos ecosistemas y hábitats, protegiéndolos y promoviéndoles para difundir sus estudios y conocimientos.

También existen constructoras interesadas en urbanizar gran parte de la reserva, lo cual propicia la paulatina desaparición de la reserva y afecta seriamente las conectividades de los ecosistemas, generando la desaparición forzosa de especies nativas, humedales, aguas subterráneas y bosque.

De esta manera se presentan tres escenarios alternativos de solución para el replanteamiento y rehabilitación de la reserva.

a. Conservación total sin intervención. Al mantener la reserva en su estado actual se continuaría con la pérdida de ecosistemas y de especies que allí habitan, esto debido a que no existe una conectividad ambiental con ningún otro hábitat, es decir este se encuentra aislado. Además, los humedales están contaminados y la presencia mayoritaria de viveros contaminan de igual forma las aguas subterráneas.

b. Urbanización total de la reserva. En este escenario se plantea una reserva fraccionada. Una propuesta que traería la típica contaminación y destrucción por parte de los humanos lo cual 
haría desaparecer los humedales, erosionaría la tierra y finalmente reduciría las conectividades y por lo tanto generaría una pérdida parcial de las diferentes especies nativas de la región. Esta es una propuesta de la alcaldía local.

c. Rehabilitación de la reserva. El proyecto de rehabilitación integral de la Reserva, busca desarrollar un método de conectividad ambiental que propicie espacios de encuentros, desarrollo económico y social donde exista un beneficio mutuo entre todos los actores involucrados. Esta propuesta fortalece la existencia de ecosistemas actuales, para atraer diferentes especies endémicas y migratorias. Propuesta desarrollada en el presente documento.

A partir de la investigación se determina que los escenarios 1 y 2 , planteados de acuerdo a las posibilidades actuales, no son adecuados para la reserva, dejando como opción su la cual permitiría prolongar la existencia de las diferentes especies ambientales que habitan allí, y permitiendo que la ciudad cuente con una gran zona de protección, beneficiando el medio ambiente y sus ecosistemas, como también asegurar la conexión de los diferentes actores, permitiendo una relación entre el ser humano y la naturaleza, generando conciencia ambiental.

\section{Plan propuesto para la rehabilitación}

A continuación, a partir de los resultados obtenidos en la investigación, se propone un nuevo modelo de ocupación dentro de la reserva van der Hammen, que no sólo atenderá las variables de movilidad, sino también las variables de conectividad de los distintos ecosistemas, parches y bordes, dejando muestra de los usos anteriores para la memoria de la vocación productiva del lugar.

Para llevar a cabo la propuesta de rehabilitación de la reserva, desde el tema de movilidad se contempla el diseño de vías aéreas para la protección de los recursos naturales, sin embargo, de esas vías transitadas se deben clasificar las vías diseñando unas exclusivas para el trasporte público y otras mixtas como se muestra en la figura 1.

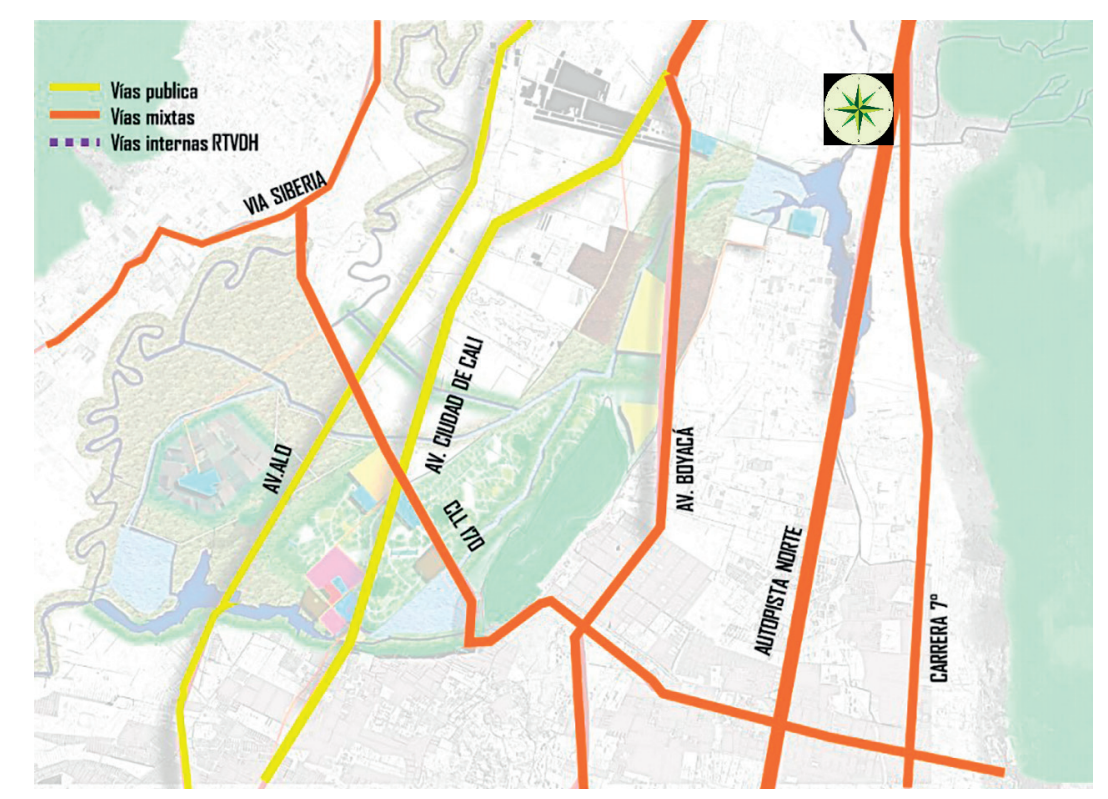

Figura 1. Mapa de las vías principales reserva Thomas van der Hammen.

Nota: las vías que se plantea dejar dentro de la Reserva deben tener un control específico, para que no afecte los ecosistemas. 
Para el desarrollo total del proyecto de rehabilitación de la reserva se plantea en tres fases de intervención, cada una de las cuales define un espacio temporal específico para su realización. Las fases de intervención son las siguientes:

Fase 1. Es una fase a un plazo corto de 7 años y contará con el adelanto de los siguientes parches dentro del proyecto: parche 1: Protección borde de la reserva, parche 2: descontaminación del humedal de La Conejera, conexión con la Salitrosa y ubicar humedales artificiales en zonas cercanas a las fuentes hídricas principales, parche 3: mitigar fuentes contaminantes en el agua subterránea (zona viveros), parche 4: densificar zona de viveros, parche 5: Reforestación del bosque de las Mercedes en dirección al río Bogotá y conexión con humedal de la conejera, parche 6: propiciar zonas de agricultura considerando buenas prácticas agrícolas, parche 7: construcción de centro de investigación científica y el parche 8: construcción de cerca viva.

Fase 2. La fase segunda es una fase a un plazo mediano de 25 años y contara con el adelanto de los siguientes parches dentro del proyecto: parche 1: conexión del humedal de Torca y Guaymaral con la Conejera a través de senderos verdes para recreación pasiva, parche 2: cerca viva entre las zonas del bosque nativo y bosque con senderos, parche 3: reforestar zona restante de viveros, parche 4: reforestar parte del terreno frente al cerro de La Conejera generando senderos de interpretación, parche 5: construcción de un jardín botánico que apoye a la conservación del bosque y de especies, y parche 6: potenciar la agricultura con buenas prácticas agrícolas.

Fase 3. La fase segunda es una fase a un plazo mediano de 25 años y contara con el adelanto de los siguientes parches dentro del proyecto: parche 1: realizar un museo de historia natural aprovechando la infraestructura existente, parche 2: diseñar nuevos puntos de descargue de agua subterránea y humedales artificiales, parche 3: reforestar borde de los humedales conectando con los cerros orientales y parche 4: generar puntos de protección de bosque en conexión con los cerros orientales. Además de realizar la conectividad con fases anteriores.

\section{Plan maestro: intersticio ecorrelacional}

Para el desarrollo de la primera fase de rehabilitación de la reserva, se plantea un nuevo sistema de ocupación que se soporta en la conectividad ecorrelacional, este sistema refuerza la estructura natural de inicio presente en la zona, así como una nueva forma de ocupación de los viveros, a fin de dejar una huella de los mismos en la reserva, por último propone espacios de investigación científica y zonas de avistamiento de aves a fin de propiciar un mercado de turismo ecológico que permita que la reserva autogestiones sus ingresos. A continuación se expone la nueva forma de ocupación de la reserva en la fase 1.

- Densificación de los viveros. Con respecto a las alternativas de viveros y a la innovación o aplicación de nuevos métodos de construcción de estos, se plantea una densificación de los viveros, es decir, la reducción de área o de zonas plantadas de viveros. Esto se lograría con la construcción de viveros verticales.

Se muestra un plan maestro (ver figura 3) de cómo se puede desarrollar un nuevo modelo de ocupación para las empresas que existen dentro de la reserva. La cantidad de edificios de viveros que se desarrollara en el plan maestro, dependerá de la capacidad de recolección de agua lluvia que tenga las hectáreas a desarrollar. 


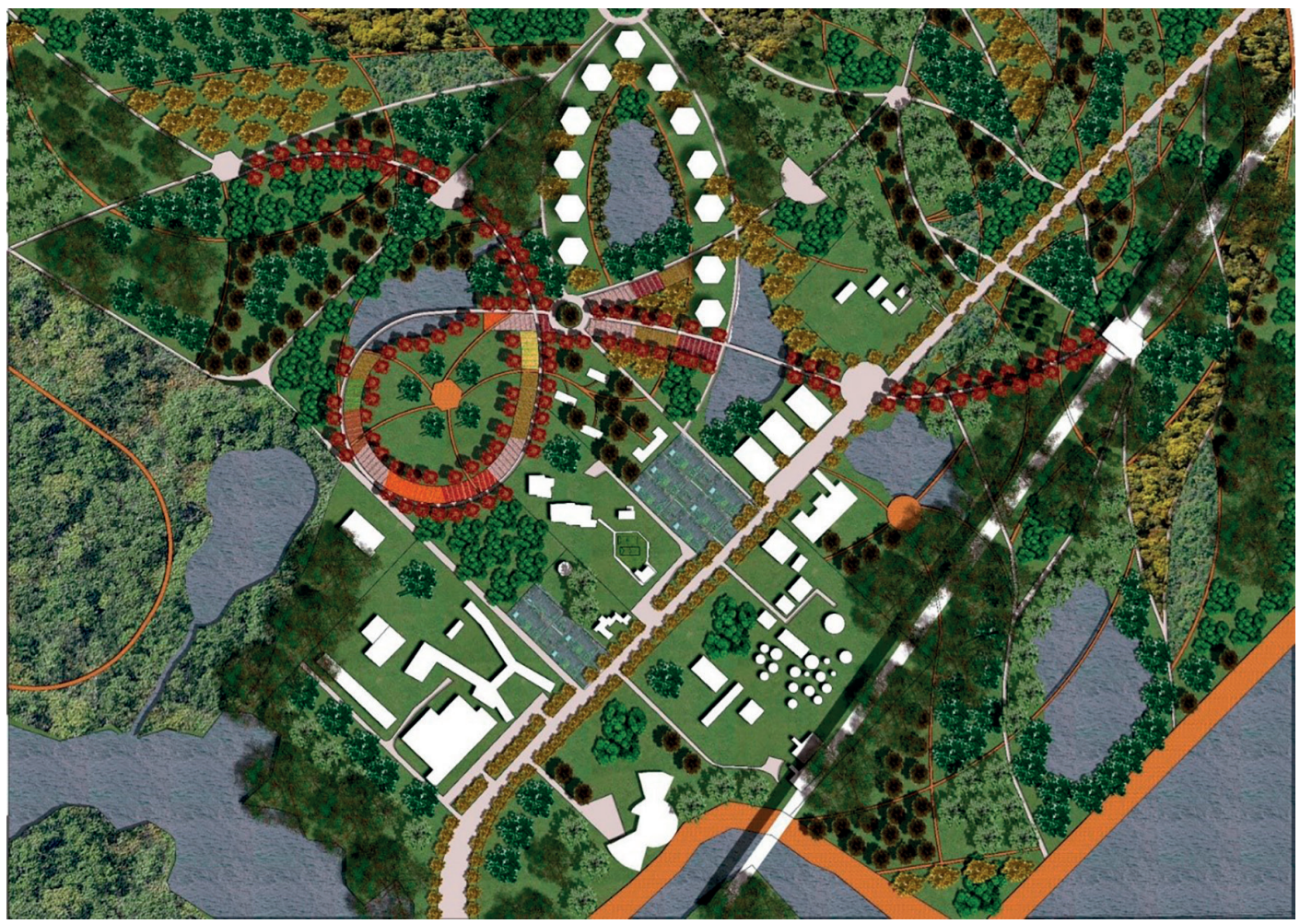

Figura 2. Plan Maestro.

Nota: En el plan parcial se tiene en cuenta las diferentes especies de vegetación nativa de Bogotá, Recuperando los bosques, los humedales, y permitiendo modificar los métodos de cultivo.

- Atracción turística. La manera en que las gentes puedan conocer e interactuar en la forma correcta, con la reserva radica principalmente en su capacidad de atracción. La atracción turística dentro de la reserva para la propuesta se compone principalmente de: restaurantes orgánicos, galerías florales, senderos ecológicos, centros de investigación, museos, entre otros (figura 4), logrando una interacción directamente positiva con los actores dentro de la reserva.

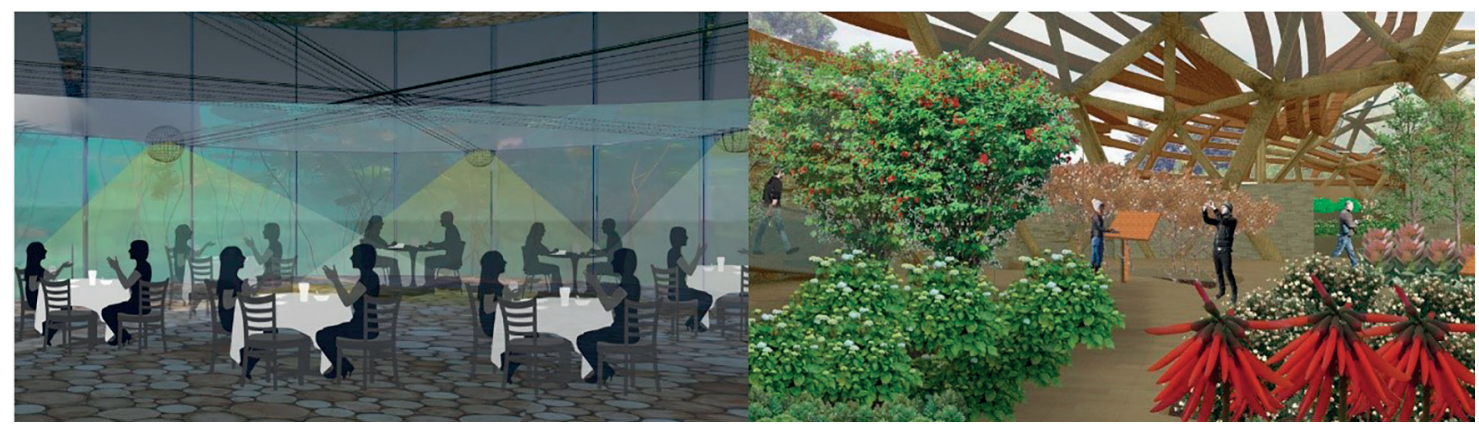

Figura 3. Atracción turística dentro de la reserva.

Nota: los espacios interiores están pensado para generar confort a los usuarios que visitan este proyecto, permitiendo que se puedan experimentar diferente ambiente en un mismo lugar. 
- Descontaminación. El impacto con la realización del proyecto debe incidir de manera positiva sobre la reserva, no solamente conservándola sino buscando alternativas o soluciones para revertir los daños causados por la contaminación. Los humedales artificiales representan una alternativa para descontaminación de las aguas en la reserva (figura 5).

La idea de los humedales artificiales consta en un sistema de filtrado entre los humedales, es decir, el agua que viene del humedal de Torca y Guaymaral, estará pasando por un primer humedal artificial que hará el proceso de descontaminación y, posteriormente, se conectará con el humedal de La Conejera. Igualmente, la quebrada la Salitrosa se descontaminará con un segundo humedal artificial y su flujo retornaría al humedal de La Conejera. De igual forma este humedal contendrá un tercer humedal artificial, donde finalmente el agua limpia desembocará en el río Bogotá. El tiempo estimado de descontaminación por humedal es de aproximadamente 20 años.

\section{Ilación Ambiental}

El proyecto Ilación Ambiental es un proyecto que pretende establecer un lugar donde se pueda interactuar con la naturaleza y conocer los ciclos ambientales locales, conexiones entre hábitats, procesos de producción de rosas, manejo y descontaminación de humedales y de aguas en general, procesos de cultivos orgánicos y alimentación saludable; este lugar pretende ser un espacio educativo con la razón de generar consciencia ecológica entre los usuarios. Los espacios que se presentan dentro del proyecto se enumeran a continuación:

\section{Vivero}

Viveros de forma vertical quienes densificaran las áreas ocupadas por su mismo establecimiento y ayudara en la producción de las flores contando como principal fuente de abastecimiento el agua pluvial con un sistema de recolección de aguas lluvia.

\section{Pabellón ambiental}

Se propone unas zonas de exhibición de los bosques nativos de la reserva que estaría disponible durante todo el año; contará además con una sala de información histórica sobre la reserva; espacio para interactuar con las entidades de protección animal a especies en peligro de extinción; un centro médico; y por ultimo un espacio especializado en la investigación de la ecología de la reserva.

\section{Cultivos orgánicos y restaurante subacuático}

Se pretende crear espacios armónicos entre sus independencias, es decir, respecto a un restaurante orgánico, se pretende extraer productos desde los cultivos orgánicos, generados en la reserva, para preparar los alimentos dentro del restaurante.

Haciendo referencia al restaurante, este tendrá una capacidad de recibir un total de 117 personas en su cupo total. Presentará dos escenarios ambientalmente pensados, el primero consta de un restaurante subacuático, con un observatorio de los ecosistemas acuáticos del sitio; y el segundo escenario es presentar al visitante la posibilidad de interactuar con entornos ambientales y propios de la vida campesina, es decir, la posibilidad de interactuar con dichos cultivos orgánicos.

\section{Cápsulas de cultivos de fitoplancton}

Este espacio estaría interactuando directamente con el humedal como se muestra en la figura 6. En este lugar se podrá observar el proceso de descontaminación del agua del humedal presentado una 
propuesta educativa para las nuevas generaciones en temas relacionados con el proceso de tratamiento por balsas Vetiver y cultivos de fitoplancton.

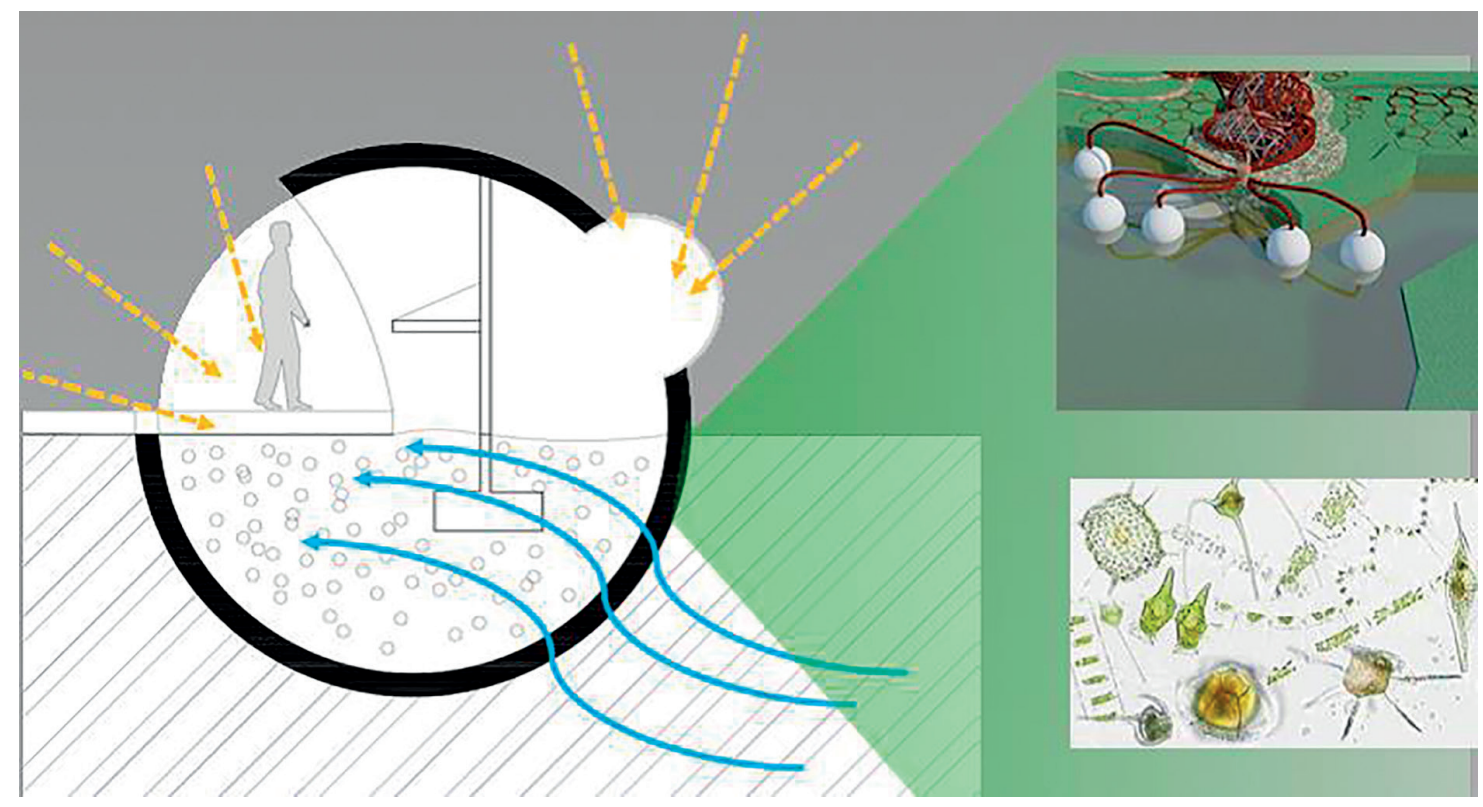

Figura 4. Cápsulas subacuáticas.

Nota: las capsulas tendrán la opción de que el usuario pueda conocer nuevos métodos de descontaminación del agua, como es el caso del tratamiento por fitoplancton, que se localiza por todo el eje del recorrido del agua.

Además de contar con otros espacios como una zona administrativa, producción de rosas, observatorio de aves, a continuación, se muestra una descripción general del edificio (tabla 1).

Tabla 1. Características de la construcción

\begin{tabular}{lr}
\hline \multicolumn{1}{c}{ Número de niveles } & \multicolumn{1}{c}{$\mathbf{4}$} \\
\hline Límite de lote & $20000 \mathrm{~m}^{2}$ \\
Índice de construcción & $1650 \mathrm{~m}^{2}$ \\
Índice de ocupación & $13 \%$ \\
Planta primer nivel & $650 \mathrm{~m}^{2}$ \\
Planta segundo nivel & $610 \mathrm{~m}^{2}$ \\
Planta tercer nivel & $236 \mathrm{~m}^{2}$ \\
Planta cuarto nivel & $150 \mathrm{~m}^{2}$ \\
\hline
\end{tabular}

\section{Conclusiones}

Las actuales condiciones de la Reserva Thomas van der Hammen, vienen debilitando las acciones en pro de la conectividad integral necesarias en el sector norte de la ciudad. Estas condiciones ocasionadas por los modos de consumo del suelo, especialmente el uso de los viveros, deben revertirse en acciones sistémicas integrales que busquen un equilibrio posible entre los distintos actores en y propendan por una meta común que esté soportada por el beneficio ambiental competitivo. 
Una evidencia de la pérdida de conectividad son las fragmentaciones espaciales de los parches en los ecosistemas presentes, lo cual lleva a reducciones de parte de la flora y la fauna, exponiendo a algunas especies endógenas al peligro de extinción. Paralelo a ello se encuentra que los procesos de contaminación hídrica tienen diversos orígenes, sin embargo, se destaca el manejo de viveros que utilizan fertilizantes y fungicidas, los cuales por la forma de uso y composición, percolan al subsuelo siendo la mayor carga contaminante de las reservas hídricas.

Bajo estas y otras consideraciones, se plantea el proyecto de rehabilitación de la reserva, pensado para brindar un beneficio ambiental, que no solo podría potenciar el desarrollo de la ciudad en su calidad socio-espacial, sino también en su calidad ambiental y económica, al revaluar las formas de ocupación actual con nuevas posibilidades espaciales que permitan incrementar el turismo cultural y ecológico del Distrito con una oferta de alto impacto local y regional especialmente, que propenda por vincular a todos los actores presentes de forma equilibrada.

El plan maestro presentado está enfocado en el cambio de los métodos de cultivo de los floricultores, desde la opción de un cultivo en vertical con aprovechamiento del agua lluvia y sin desmejora de la producción. Aunado a los viveros se refuerzan las áreas blandas con especies de fauna y flora endémicas, propiciando un fortalecimiento de los ecosistemas y de las especies actuales; se proponen espacios de avistamiento de aves y de investigación científica especializada.

La investigación determina así que es posible brindar espacios naturales de coexistencias entre especies humanas y no humanas, que de forma competitiva propendan por crear escenarios futuros, que le brinden al Distrito y a los municipios aledaños oportunidades viables y equilibradas de planeación territorial.

\section{REFERENCIAS}

CAR. (2011). Acuerdo 11. Bogotá: Diario Oficial 48.156.

CAR. (2014). Acuerdo 201. Bogotá: Diario Oficial 49.317.

Connor, E., Courtney, A. y Yoder, J. (2000). Individuals-area relationships. The relationships between animal population density and area. Ecology, 81(3), 734-748.

Dudley, N. (2008). Directrices para la aplicación de las categorías de gestion en áreas protegidas. Suiza: UICN, Gland, Suiza.

Gurrutxaga, M., y Lozano, P. J. (2006). Efectos de la fragmentación de hábitats y pérdida de conectividad ecológica dentro de la dinámica territorial. Revista de Geografía, 16, 35-54.

Universidad Autónoma de Ciudad Juárez. (2013). Introducción en áreas naturales protegidas (Hoja técnica de divulgación científica). México: Universidad Autónoma de Ciudad Juárez.

Londoño, J. (2012). Discusiones sobre la presencia de aves rapaces, aves migratorias y aves bajo algún grado de amenaza en la ciudad de Pereira. Revista Luna Azul, 36, 134-164.

Molina, L., y Osorio, J. (1995). Guía de Aves de Santa fe Bogotá. Bogotá: DAMA.

Pulliman, H. (1988). Sources, sinks and population regulation. The American Naturalist, 132(5), 652-661.

Remolina, F., y Chisaca, L. (2012). Importancia de la conectividad ecológica como herramienta de planificación de conservación en áreas rurales. Bogotá: Secretaria Distrital del Medio Ambiente y Dirección de Ambiente y Ruralidad. 
Ruiz, T., Otero, G., Ramires, A., y Trespalacioas, G. (2008). Biodiversidad y conectividad esológica en la localidad de Suba. Bogota D. C.: Alexander von Humbolt.

Sepulveda, , Moreira, A., y Villarroel, P. (1997). Conservación biológica fuera de las áreas silvestres protegidas. Ambiente y Desarrollo, 13(2), 48-58.

Tolón, A., y Lastra, X. (2008). Los espacios naturales protegidos. Concepto, evolución y situación actual en España.Revista Electronic@ de Medioambiente UCM, 5,1-25. https://www.ucm.es/ data/cont/media/www/pag41228/ART\%20A.TOLON\%20X.\%20LASTRA.pdf

WWF. (2015). Buenas prácticas para la definición de redes ecológicas en España. Conectividad Ecológica: importancia, situación en España y criterios para identificar redes ecológicas. Recuperado de WWF, 1-11. http://awsassets.wwf.es/downloads/buenas_practicas_para_la_definicion_de_ redes_ecologicas_en_espana_gr.pdf 